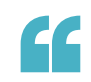

The dODN can elicit a twopronged effect

\title{
HAEMATOLOGICAL CANCER Send in the decoys, STAT!
}

STAT3 is a transcription factor involved in cell survival and immunosuppression, and is implicated in acute myeloid leukaemia (AML). Efforts to inhibit STAT3 have been limited by the inherent difficulties in targeting transcription factors lacking catalytic activity; however, Marcin Kortylewski and co-workers have developed a cell-selective STAT3 decoy oligodeoxynucleotide (dODN) that overcomes these challenges.

The dODN comprises STAT3 binding and CpG DNA sequences. CpG motifs are recognized by Tolllike receptor 9 (TLR9) enabling targeting of the dODN to myeloid cells that express this receptor, including AML cells, tumour-associated macrophages, and myeloid-derived suppressor cells (MDSCs). Indeed, the dODN efficiently penetrated all AML cell types (including leukaemic stem/progenitor cells), sequestered STAT3, inhibited its transcriptional activity, and caused disease regression in mouse models of human AML.

Treatment of primary human TLR9 ${ }^{+}$AML cells with dODN increased their immunogenicity and decreased expression of the immunosuppressive enzyme arginase- 1 . In imunocompetent mice, dODN increased effector-T-cell infiltration into leukaemia reservoirs, decreased MDSC and regulatory-T-cell infiltration, and could completely eradicate AML in immunocompetent mice.

"Blocking STAT3 unleashes TLR9triggered immune responses, resulting in gain-of-function effects that augment both the efficacy and potency of the dODN," Kortylewski explains. "The dODN can elicit a two-pronged effect - direct cytotoxicity and antileukaemic immune responses; however, the immune effects alone might be sufficient for elimination of established AML by releasing the breaks on $\mathrm{CD}^{+} / \mathrm{CD} 4^{+} \mathrm{T}$-cell-mediated immunity."

Kortylewski concludes, "owing to the role of STAT3 as a major regulator of tumour immune evasion, this approach might be broadly applicable to immunotherapy of malignancies other than AML." Furthermore, the development of similar dODN targeting other oncogenic transcription factors might enable translation of this strategy to other cancer types.

\section{David Killock}

ORIGINAL ARTICLE Zhang, Q. et al.

Serum-resistant CPG-STAT3 decoy for targeting survival and immune checkpoint signaling in acute myeloid leukemia. Blood http://dx.doi. org/10.1182/blood-2015-08-665604 (2016) 